\title{
Rings of PDE-preserving operators on nuclearly entire functions
}

\author{
by \\ Henrik Petersson (Göteborg)
}

\begin{abstract}
Let $E, F$ be Banach spaces where $F=E^{\prime}$ or vice versa. If $F$ has the approximation property, then the space of nuclearly entire functions of bounded type, $\mathscr{H}_{\mathrm{Nb}}(E)$, and the space of exponential type functions, $\operatorname{Exp}(F)$, form a dual pair. The set of convolution operators on $\mathscr{H}_{\mathrm{Nb}}(E)$ (i.e. the continuous operators that commute with all translations) is formed by the transposes $\varphi(D) \equiv{ }^{t} \varphi, \varphi \in \operatorname{Exp}(F)$, of the multiplication operators $\varphi: \psi \mapsto \varphi \psi$ on $\operatorname{Exp}(F)$. A continuous operator $T$ on $\mathscr{H}_{\mathrm{Nb}}(E)$ is PDE-preserving for a set $\mathbb{P} \subseteq \operatorname{Exp}(F)$ if it has the invariance property: $T \operatorname{ker} \varphi(D) \subseteq \operatorname{ker} \varphi(D), \varphi \in \mathbb{P}$. The set of PDE-preserving operators $\mathscr{O}(\mathbb{P})$ for $\mathbb{P}$ forms a ring and, as a starting point, we characterize $\mathscr{O}(\mathbb{H})$ in different ways, where $\mathbb{H}=\mathbb{H}(F)$ is the set of continuous homogeneous polynomials on $F$. The elements of $\mathscr{O}(\mathbb{H})$ can, in a one-to-one way, be identified with sequences of certain growth in $\operatorname{Exp}(F)$. Further, we establish a kernel theorem: For every continuous linear operator on $\mathscr{H}_{\mathrm{Nb}}(E)$ there is a unique kernel, or symbol, and we characterize $\mathscr{O}(\mathbb{H})$ by describing the corresponding symbol set. We obtain a sufficient condition for an operator to be PDE-preserving for a set $\mathbb{P} \supseteq \mathbb{H}$. Finally, by duality we obtain results on operators that preserve ideals in $\operatorname{Exp}(F)$.
\end{abstract}

1. Introduction. The notion of "PDE-preserving" was originally introduced by Calvi and Filipsson (see [4, Paper II]). They defined so-called PDE-preserving projectors on the space of entire functions in $d$ variables (onto polynomial spaces). We extend the definition and the study to infinitedimensional holomorphy (see also [7-10]): A continuous operator $T$, on the space $\mathscr{H}_{\mathrm{Nb}}=\mathscr{H}_{\mathrm{Nb}}(E)$ of nuclearly entire functions of bounded type on a Banach space $E$, is $P D E$-preserving for a given set of convolution operators when the kernel set for every convolution operator in the set is invariant un$\operatorname{der} T$ (see also Definition 1, where we use the fact that we can identify the set $\mathscr{C}=\mathscr{C}(E)$ of convolution operators on $\mathscr{H}_{\mathrm{Nb}}$ with the space of exponential type functions $\operatorname{Exp}=\operatorname{Exp}(F)$ defined on the dual, or predual, $F$ of $E$, in a one-to-one way, $\operatorname{Exp} \ni \varphi \mapsto \varphi(D) \in \mathscr{C}$, and use a slightly different terminol-

2000 Mathematics Subject Classification: Primary 46G20, 47L10, 47A15; Secondary 47B99, 47F05.

Research supported by the The Royal Swedish Academy of Sciences. 
ogy). (A convolution operator is a continuous operator that commutes with all translations.) We note that the set of PDE-preserving operators, for a given set, forms a ring under composition, and $\mathscr{C}$ is a commutative subring. Further, $\mathscr{H}_{\mathrm{Nb}}$ is a module over any ring of PDE-preserving operators.

It follows that an operator is PDE-preserving for the entire set $\mathscr{C}$ iff it is itself a convolution operator (Proposition 1). A fundamental, and main, result is Theorem A where we establish a one-to-one correspondence between the ring $\mathscr{O}$ of PDE-preserving operators for the set $\mathscr{C}_{\mathrm{H}}=\mathscr{C}_{\mathrm{H}}(E)$ of homogeneous convolution operators (i.e. the differential operators $P(D)$ where $P$ is a continuous homogeneous polynomial) and a set of sequences in Exp. In fact, in total we obtain three different such one-to-one correspondences, described in Theorems A, B and C, where Theorems B and C also yield the following. We prove that every continuous operator on $\mathscr{H}_{\mathrm{Nb}}$ can be written as an infinite type differential operator $P(\cdot, D)$ with variable coefficients with a unique symbol $P(x, y)$ (Proposition 2), and in Theorem B we describe the symbols for the PDE-preserving operators in $\mathscr{O}$. Theorem $\mathrm{C}$ shows that every operator $T \in \mathscr{O}$ can be expanded in powers of the Euler operator, which is the analogue of the operator $x_{1} \partial / \partial x_{1}+\cdots+x_{d} \partial / \partial x_{d}$ when $E=\mathbb{C}^{d}$, with convolution operators as coefficients.

All these characterization results of $\mathscr{O}$ suggest a study of the set of PDEpreserving operators for any given set in $\mathscr{C}$ containing the homogeneous convolution operators (in this way we deal with rings of infinite type differential operators with variable coefficients and thus with various $\mathcal{D}$-module structures [1] on $\mathscr{H}_{\mathrm{Nb}}$ ). We obtain a sufficient condition on the sequence in Exp, representing a PDE-preserving operator $T$ for $\mathscr{C}_{\mathrm{H}}$, in order that $T$ is PDE-preserving for a larger set (Theorem 1).

A central role in our study is played by the Martineau duality: $\mathscr{H}_{\mathrm{Nb}}$ forms a dual pair with the space, and ring, of exponential type functions Exp. The orthogonal complement of the kernel of any convolution operator $\varphi(D)$ is the closed principal ideal $\operatorname{Im} \varphi=\operatorname{Exp} \cdot \varphi$ in Exp. By duality arguments, the operator $T$ on $\mathscr{H}_{\mathrm{Nb}}$ is PDE-preserving, for a given set of convolution operators, if and only if the transpose ${ }^{t} T$ is ideal-preserving in the sense that every corresponding ideal $\operatorname{Im} \varphi$ is invariant under ${ }^{t} T$. From this we deduce that an operator is ideal-preserving for a set $\supseteq \mathscr{C}_{\mathrm{H}}$ iff it is the transpose of some PDE-preserving operator for the same set (Theorem 2). Thus, by our characterization of rings of PDE-preserving operators, we canonically describe rings of ideal-preserving operators.

The article is organized as follows. In the next section we introduce some notation and definitions. In particular, we introduce the space of nuclearly entire functions, referring to $[5,6]$ for a comprehensive exposition of the theory of this space (see also [3]). Further, as an introduction, we prove the 
elementary result that the convolution operators are the only operators that are PDE-preserving for the entire set $\mathscr{C}$. In Section 3 we prove Theorems A, $\mathrm{B}$ and $\mathrm{C}$, i.e. we characterize the PDE-preserving operators for $\mathscr{C}_{\mathrm{H}}$. In Section 4 we obtain a sufficient condition for an operator to be PDE-preserving for a given set containing $\mathscr{C}_{\mathrm{H}}$. In the last Section 5 , we pass to duality and show how to obtain ideal-preserving operators.

Analogues of Theorems A, B and C are obtained in [10] for the finitedimensional case. (In general, [10] may be a nice complement to this paper for a non-expert in infinite-dimensional holomorphy.) Theorem A can be found in [8] and is obtained here with a different technique. The proof in [8] is more straightforward while the one given here is more informative. Our results in Section 4, i.e. on PDE-preserving operators for sets containing the homogeneous convolution operators, are new even for finite-dimensional holomorphy. When $E$ is finite-dimensional, the topology on $\mathscr{H}_{\mathrm{Nb}}(E)$ coincides with the compact-open topology. Thus, in this case, $\mathscr{H}_{\mathrm{Nb}}$ is reflexive and the main result in Section 5, Theorem 2, follows by simple duality arguments as noted in [10]. For results on PDE-preserving projectors, for different infinite-dimensional holomorphy types, we refer to $[7,8]$. (In [7] we deal with the Hilbert-Schmidt holomorphy type and remark that most of the results in this paper can presumably be extended to this holomorphy type, or more generally, to any holomorphy type for which there exists a Martineau duality (1).)

2. Notation and preliminaries. A Banach pairing is a pairing $(E, F)$ where $E$ and $F$ are (complex) Banach spaces and where $F=E^{\prime}$ or the other way around. (Thus the norm topologies on $E$ and $F$ are the strong topologies $\beta(E, F)$ and $\beta(F, E)$ respectively.) Let $(E, F)$ be a fixed Banach pairing. By $\mathscr{P}\left({ }^{n} F\right)$ we denote the Banach space of continuous $n$-homogeneous polynomials on $F$ equipped with the usual norm which we denote by $\|\cdot\|_{n}$. The set of continuous homogeneous polynomials, $\bigcup_{n \in \mathbb{N}} \mathscr{P}\left({ }^{n} F\right)$, is denoted by $\mathbb{H}(F)$. $\mathscr{P}_{\mathrm{N}}\left({ }^{n} E\right)$ denotes the Banach space of nuclear $n$-homogeneous polynomials on $E$, i.e. the space of all polynomials $P$ of the form

$$
P=\sum_{k=1}^{\infty}\left\langle\cdot, y_{k}\right\rangle^{n}, \quad\left(\left\|y_{k}\right\|^{n}\right) \in \ell_{1},
$$

where $y_{k}$ are vectors in $F$, provided with the nuclear norm $\|\cdot\|_{n}$. (For simplicity we do not specify $F$ in the notation $\mathscr{P}_{\mathrm{N}}\left({ }^{n} E\right)$.) Thus the set of finite type polynomials on $E$, i.e. the subspace of $\mathscr{P}_{\mathrm{N}}\left({ }^{n} E\right)$ spanned by the polynomials of the form $\langle\cdot, y\rangle^{n}, y \in F$, is dense in $\mathscr{P}_{\mathrm{N}}\left({ }^{n} E\right)$. If $F$ has the approximation property, the map $\mathcal{F}_{n}$ defined by $\mathcal{F}_{n} \lambda(y) \equiv \lambda\langle\cdot, y\rangle^{n}$ is a topological isomorphism between $\mathscr{P}_{\mathrm{N}}^{\prime}\left({ }^{n} E\right)$ and $\mathscr{P}\left({ }^{n} F\right)$ (see $\left.[3,5,6,9]\right)$. In this way $\mathscr{P}_{\mathrm{N}}\left({ }^{n} E\right)$ and $\mathscr{P}\left({ }^{n} F\right)$ are in duality by $\langle P, Q\rangle_{n} \equiv \mathcal{F}_{n}^{-1} Q(P)$ where $P \in \mathscr{P}_{\mathrm{N}}\left({ }^{n} E\right)$ and 
$Q \in \mathscr{P}\left({ }^{n} F\right)$. In particular, $\left\langle P,\langle x, \cdot\rangle^{n}\right\rangle_{n}=P(x)$ for all $P \in \mathscr{P}_{\mathrm{N}}\left({ }^{n} E\right)$ and $x \in E$ and we obtain the value at $y \in F$ for any $Q \in \mathscr{P}\left({ }^{n} F\right)$ analogously.

If $(E, F)$ is a Banach pairing, $\mathscr{H}_{\mathrm{Nb}}(E)$ is the space of all Gateaux holomorphic functions $f \in \mathscr{H}_{\mathrm{G}}(E)$ on $E$ such that $f_{n} \equiv D_{(\cdot)}^{n} f(0) / n ! \in \mathscr{P}_{\mathrm{N}}\left({ }^{n} E\right)$, $n=0,1, \ldots$, and

$$
\|f\|_{\mathrm{N}: r} \equiv \sum_{n=0}^{\infty} r^{n}\left\|f_{n}\right\|_{n}<\infty, \quad r>0
$$

equipped with the seminorms thus defined. Here $D_{a}^{n}$ denotes the $n$th directional derivative along $a$. The space $\mathscr{H}_{\mathrm{Nb}}(E)$, i.e. the nuclearly entire functions of bounded type, is a Fréchet space, and $f=\sum f_{n}$ in $\mathscr{H}_{\mathrm{Nb}}(E)$ for every $f \in \mathscr{H}_{\mathrm{Nb}}(E)$. Given $r>0, \operatorname{Exp}_{r}(F)$ denotes the Banach space of functions $\varphi \in \mathscr{H}_{\mathrm{G}}(F)$ such that, for some $M>0,|\varphi(y)| \leq M e^{r\|y\|}$, equipped with the norm

$$
\|\varphi\|_{\mathrm{E}: r} \equiv \sup _{y \in F}|\varphi(y)| e^{-r\|y\|}
$$

The space of exponential type functions on $F, \operatorname{Exp}(F)$, is the union $\operatorname{Exp} \equiv$ $\bigcup_{r>0} \operatorname{Exp}_{r}$ provided with the corresponding inductive locally convex topology. By Cauchy's estimates, it is easily checked that $\varphi \in \mathscr{H}_{\mathrm{G}}(F)$ belongs to $\operatorname{Exp}(F)$ if and only if $\varphi_{n} \equiv D_{(\cdot)}^{n} \varphi(0) / n ! \in \mathscr{P}\left({ }^{n} F\right), n=0,1, \ldots$, and $\lim \sup \left[n !\left\|\varphi_{n}\right\|_{n}\right]^{1 / n}<\infty$. Moreover, the elements of $\operatorname{Exp}(F)$ are continuous on $F$. For any $n \geq 0, H_{n}$ denotes the projector $\operatorname{Exp}(F) \rightarrow \mathscr{P}\left({ }^{n} F\right)$ defined by $\varphi \mapsto \varphi_{n}$. We use the same symbol for the corresponding projector of $\mathscr{H}_{\mathrm{Nb}}(E)$ onto $\mathscr{P}_{\mathrm{N}}\left({ }^{n} E\right)$.

By the duality between $\mathscr{P}_{\mathrm{N}}\left({ }^{n} E\right)$ and $\mathscr{P}\left({ }^{n} F\right)$, when $F$ has the approximation property, the Fourier-Borel transform $\mathcal{F}$, defined by $\mathcal{F} \lambda(y) \equiv \lambda\left(e_{y}\right)$ where $e_{y} \equiv e^{\langle\cdot, y\rangle} \in \mathscr{H}_{\mathrm{Nb}}(E)$ (similarly we let $e_{x} \equiv e^{\langle x, \cdot\rangle} \in \operatorname{Exp}(F)$ when $x \in E$ ), is an isomorphism between $\mathscr{H}_{\mathrm{Nb}}^{\prime}(E)$ (strong topology) and $\operatorname{Exp}(F)$, and we put $\mathscr{H}_{\mathrm{Nb}}(E)$ and $\operatorname{Exp}(F)$ in (Martineau) duality by

$$
\langle f, \varphi\rangle \equiv \mathcal{F}^{-1} \varphi(f)=\sum_{n=0}^{\infty} n !\left\langle f_{n}, \varphi_{n}\right\rangle_{n}, \quad f \in \mathscr{H}_{\mathrm{Nb}}(E), \varphi \in \operatorname{Exp}(F) .
$$

It is convenient to note that if $\varphi \in \operatorname{Exp}_{r}(F)$, then $\left\|\varphi_{n}\right\|_{n} \leq\|\varphi\|_{\mathrm{E}: r}(e r)^{n} / n$ ! and thus

$$
|\langle f, \varphi\rangle| \leq\|f\|_{\mathrm{N}: e r}\|\varphi\|_{\mathrm{E}: r}
$$

for all $f \in \mathscr{H}_{\mathrm{Nb}}(E)$.

From now on we let $(E, F)$ be a fixed Banach pairing where $F$ has the approximation property, and we denote by $\mathscr{L}$ the set, and ring (under composition), of continuous linear operators on $\mathscr{H}_{\mathrm{Nb}}(E)$.

Every multiplication operator $\varphi: \psi \mapsto \varphi \psi, \varphi \in \operatorname{Exp}(F)$, is weakly continuous on $\operatorname{Exp}(F)$ for the duality between $\mathscr{H}_{\mathrm{Nb}}(E)$ and $\operatorname{Exp}(F)$. The set 
of convolution operators on $\mathscr{H}_{\mathrm{Nb}}(E), \mathscr{C}=\mathscr{C}(E)$, is formed by the operators $\varphi(D) \equiv{ }^{t} \varphi: \mathscr{H}_{\mathrm{Nb}}(E) \rightarrow \mathscr{H}_{\mathrm{Nb}}(E), \varphi \in \operatorname{Exp}(F)$.

Definition 1. A continuous linear operator $T: \mathscr{H}_{\mathrm{Nb}}(E) \rightarrow \mathscr{H}_{\mathrm{Nb}}(E)$ is $P D E$-preserving for a set $\mathbb{P} \subseteq \operatorname{Exp}(F)$ if $T \operatorname{ker} \varphi(D) \subseteq \operatorname{ker} \varphi(D)$ (i.e. $\operatorname{ker} \varphi(D)$ is invariant under $T$ ) for all $\varphi \in \mathbb{P}$. The set of PDE-preserving operators for $\mathbb{P}$ is denoted by $\mathscr{O}(\mathbb{P})$.

$\mathscr{O}(\mathbb{P})$ is a ring under composition, and since any two convolution operators commute, $\mathscr{C}$ forms a commutative subring for any set $\mathbb{P}$. In particular, when $\mathbb{P}=\operatorname{Exp}(F)$ we have:

Proposition 1. $\mathscr{O}(\operatorname{Exp}(F))=\mathscr{C}(E)$.

Proof. Let $T \in \mathscr{O}(\operatorname{Exp}(F))$. We only have to prove that $T$ commutes with all translations $\tau_{a}, a \in E$, where $\tau_{a} f(x) \equiv f(x+a)$. Thus let $a \in E$ be arbitrary. By totality it suffices to prove that $\tau_{a} T e_{b}=T \tau_{a} e_{b}$, i.e., $\tau_{a} T e_{b}=$ $e^{\langle a, b\rangle} T e_{b}$ for any $b \in F$. Now, $D_{x}^{n}-\langle x, b\rangle^{n} \in \mathscr{C}$ and $e_{b} \in \operatorname{ker}\left[D_{x}^{n}-\langle x, b\rangle^{n}\right]$ for all $x \in E$ and $n \geq 0$. Hence, by assumption, $D_{x}^{n} T e_{b}=\langle x, b\rangle^{n} T e_{b}$ for all $x$ and $n$. This gives $T e_{b}=\sum D_{(\cdot)}^{n} T e_{b}(0) / n !=e_{b} T e_{b}(0)$. Thus $\tau_{a} T e_{b}=$ $\tau_{a} e_{b} T e_{b}(0)=e^{\langle a, b\rangle} e_{b} T e_{b}(0)=e^{\langle a, b\rangle} T e_{b}$.

Note also that, for any set $\mathbb{P}, \mathscr{H}_{\mathrm{Nb}}(E)$ is a module over $\mathscr{O}(\mathbb{P})$, and $\operatorname{ker} \varphi(D)$ forms a submodule for every $\varphi \in \mathbb{P}$.

3. The ring $\mathscr{O}(\mathbb{H})$. In this section we characterize $\mathscr{O}(\mathbb{H})$, i.e. the ring of PDE-preserving operators for the set $\mathbb{H}=\mathbb{H}(F)$ of homogeneous polynomials, in different ways.

Lemma 1. An operator $T: \mathscr{H}_{\mathrm{Nb}}(E) \rightarrow \mathscr{H}_{\mathrm{Nb}}(E)$ is continuous if and only if it has the following form: $T f=\sum_{n=0}^{\infty} T_{n} f$, where $T_{n}: \mathscr{H}_{\mathrm{Nb}}(E) \rightarrow$ $\mathscr{P}_{\mathrm{N}}\left({ }^{n} E\right)$ and for every $r>0,\left\|T_{n} f\right\|_{n} \leq N r^{n}\|f\|_{\mathrm{N}: R}$ for some $N=N_{r}$ and $R=R_{r}$. The sequence $\left(T_{n}\right)$ is unique and we write $T=\sum T_{n}$.

Proof. We prove that every operator of the described form is continuous. Let $r>0$ be arbitrary. Then, for any $f \in \mathscr{H}_{\mathrm{Nb}}(E),\|T f\|_{\mathrm{N}: r} \leq$ $\sum r^{n}\left\|T_{n} f\right\|_{n} \leq\|f\|_{\mathrm{N}: R} N \sum \delta^{n}$ for some $R, N>0$ and $\delta<1$ (depending on $r$ ).

Conversely, let $T$ be continuous and put $T_{n} \equiv H_{n} T: \mathscr{H}_{\mathrm{Nb}}(E) \rightarrow$ $\mathscr{P}_{\mathrm{N}}\left({ }^{n} E\right)$. Then $T f=\sum T_{n} f$ and, for any $s>0$, we have $T_{n} f=$ $\int_{|z|=s}\left[f(z(\cdot)) / z^{n+1}\right] d z / 2 \pi i$ for all $f \in \mathscr{H}_{\mathrm{Nb}}$. Thus, for any $s>0,\left\|T_{n} f\right\|_{n}=$ $\left\|T_{n} f\right\|_{\mathrm{N}: 1} \leq\|f\|_{\mathrm{N}: s} / s^{n}$. This gives the required estimate for $\left(T_{n}\right)$; the uniqueness of the sequence is obvious.

LEMma 2. Assume $T=\sum T_{m}: \mathscr{H}_{\mathrm{Nb}}(E) \rightarrow \mathscr{H}_{\mathrm{Nb}}(E)$ is PDE-preserving for the set $\mathscr{P}\left({ }^{n} F\right)$. Then there is a unique $\varphi \in \operatorname{Exp}(F)$ such that $T_{n}=$ $H_{n} \circ \varphi(D)$. 
Proof. For given $x \in E$ we define $T_{n: x} \in \mathscr{H}_{\mathrm{Nb}}^{\prime}$ by $T_{n: x}(f) \equiv H_{n} T f(x)=$ $T_{n} f(x)$. We prove that there are $\varphi_{n: x} \in \operatorname{Exp}, x \in E$, such that

$$
H_{n} \varphi_{n: x}(D) f(x)=T_{n: x}(f) .
$$

If $n=0$, then $\varphi_{0} \equiv \mathcal{F}\left(\delta_{0} \circ T\right)\left(\delta_{0} f \equiv f(0)\right)$ solves (3) for all $x$. Next, assume $n \geq 1$ and let $x \neq 0$. We show that $\mathcal{F} T_{n: x} \in \operatorname{Im}\langle x, \cdot\rangle^{n}=\left(\operatorname{ker} D_{x}^{n}\right)^{\perp}$. Let $f \in \operatorname{ker} D_{x}^{n}$ be arbitrary. Since $T$ is PDE-preserving for $\mathscr{P}\left({ }^{n} F\right)$, we have $D_{x}^{n} T f=0$ and hence $T_{n: x}(f)=D_{x}^{n} T f(0) / n !=0$. Thus there is a unique $\varphi_{n: x} \in$ Exp such that $\langle x, \cdot\rangle^{n} \varphi_{n: x}=n ! \mathcal{F} T_{n: x}$ and hence $H_{n} \varphi_{n: x}(D) f(x)=$ $T_{n: x}(f)$. We prove that the solutions $\varphi_{n: x}, x \neq 0$, are independent of $x$. Multiplication by $\langle x, \cdot\rangle^{n}, x \neq 0$, is one-to-one on Exp, thus it suffices to prove that

$$
\langle x, \cdot\rangle^{n} \varphi_{n: x}=\langle x, \cdot\rangle^{n} \varphi_{n: x^{\prime}}, \quad x, x^{\prime} \neq 0 .
$$

For $y \in F$ we define $u(y) \equiv\langle\cdot, y\rangle^{n} \in \mathscr{P}_{\mathrm{N}}\left({ }^{n} E\right)$ and

$$
v(y) \equiv\langle\cdot, y\rangle^{n} \varphi_{n:(\cdot)}(y)=n ! H_{n} T e_{y} \in \mathscr{P}_{\mathrm{N}}\left({ }^{n} E\right) .
$$

Assume $P \in \mathscr{P}\left({ }^{n} F\right)$ and that $P \in\{u(y)\}^{\perp}$ with respect to the duality $\langle\cdot, \cdot\rangle_{n}$ between $\mathscr{P}_{\mathrm{N}}\left({ }^{n} E\right)$ and $\mathscr{P}\left({ }^{n} F\right)$. Then $P(y)=\langle u(y), P\rangle_{n}=0$ and hence $P(D) e_{y}=P(y) e_{y}=0$. Since $T$ is PDE-preserving for $\mathscr{P}\left({ }^{n} F\right)$, we have $P(D) T e_{y}=0$, from which we deduce that $\langle v(y), P\rangle_{n}=0$. Thus $\{u(y)\}^{\perp} \subseteq$ $\{v(y)\}^{\perp}$ and consequently $v(y)=c(y) u(y)$, i.e.

$$
\langle x, y\rangle^{n} \varphi_{n: x}(y)=c(y)\langle x, y\rangle^{n}, \quad x \in E,
$$

for some constant $c=c(y)$ (independent of $x$ ). If $y_{0} \neq 0$ we deduce from (5) that $c(y)=\varphi_{n: x_{0}}(y)$ in a neighbourhood of $y_{0}$ for some $x_{0} \in E$. Thus we have a continuous map $F \backslash\{0\} \ni y \mapsto c(y)$. Let $x, x^{\prime} \neq 0$. If $\langle x, y\rangle=0$, both sides in (4) vanish, so assume $\langle x, y\rangle \neq 0$. Then $\varphi_{n: x}(y)=c(y)$, and since $y \neq 0$, there is a sequence $\left(y_{j}\right)$ in $F$ such that $y_{j} \rightarrow y$ and $\left\langle x^{\prime}, y_{j}\right\rangle \neq 0$ for all $j$. Hence, by (5) and continuity,

$$
\varphi_{n: x^{\prime}}(y)=\lim c\left(y_{j}\right)=c(y)=\varphi_{n: x}(y) .
$$

Thus $\varphi_{n}=\varphi_{n: x}$ for all $x \neq 0$ for some $\varphi_{n} \in \operatorname{Exp}$.

If $x=0$ and $n \geq 1$, both sides in (3) vanish for every function in Exp, hence for every $n \geq 0$ we have obtained a $\varphi=\varphi_{n} \in \operatorname{Exp}$ such that $H_{n} \varphi_{n}(D)=H_{n} T$.

We denote by $\Sigma=\Sigma(F)$ the set of all sequences $\left(\varphi_{0}, \varphi_{1}, \ldots\right)$ in $\operatorname{Exp}(F)$. Let $\mathcal{S}=\mathcal{S}(F)$ denote the set of all $\Phi=\left(\varphi_{n}\right) \in \Sigma$ such that, for some $r, M, N>0,\left|\varphi_{n}(y)\right| \leq N M^{n} e^{r\|y\|}$ for all $n \geq 0$ and $y \in F$.

TheOREM A. The ring $\mathscr{O}(\mathbb{H})$ consists of the operators of the form $\Phi(D) f$ $\equiv \sum H_{n} \varphi_{n}(D) f$ where $\Phi=\left(\varphi_{n}\right) \in \mathcal{S}(F)$ and is unique.

Proof. Assume $T=\sum T_{n} \in \mathscr{O}(\mathbb{H})$. By Lemma 2, $T_{n}=H_{n} \varphi_{n}(D)$, $n=0,1, \ldots$, for some unique $\Phi=\left(\varphi_{n}\right) \in \Sigma$. We have to prove that 
$\Phi \in \mathcal{S}$. From the identity $\langle x, y\rangle^{n} \varphi_{n}(y)=D_{x}^{n} T e_{y}(y)$ we deduce, by identifying homogeneous parts, $\langle x, y\rangle^{n} \varphi_{n m}(y)=\left\langle T\langle\cdot, y\rangle^{n+m},\langle x, \cdot\rangle^{n}\right\rangle /(n+m)$ !, $\varphi_{n m} \equiv H_{m}\left(\varphi_{n}\right) \in \mathscr{P}\left({ }^{m} F\right)$. If $\|x\|=1$ we obtain, by continuity and (2),

$$
\begin{aligned}
\left|\left\langle T\langle\cdot, y\rangle^{n+m},\langle x, \cdot\rangle^{n}\right\rangle\right| & \leq\left\|T\langle\cdot, y\rangle^{n+m}\right\|_{\mathrm{N}: e}\left\|\langle x, \cdot\rangle^{n}\right\|_{\mathrm{E}: 1} \\
& \leq C\left\|\langle\cdot, y\rangle^{n+m}\right\|_{\mathrm{N}: R} n ! \leq C n ! R^{n+m}\|y\|^{n+m},
\end{aligned}
$$

for some $R=R(T)$ and $C=C(T)$. If $P$ and $Q$ belong to $\mathscr{P}\left({ }^{n} F\right)$ and $\mathscr{P}\left({ }^{m} F\right)$ respectively, the estimate in $[2$, p. 72] implies

Thus,

$$
\|P\|_{n}\|Q\|_{m} \leq(2 e)^{n+m}\|P Q\|_{n+m} .
$$

$$
\begin{aligned}
\left\|\varphi_{n m}\right\|_{m} & =\left\|\langle x, \cdot\rangle^{n}\right\|_{n}\left\|\varphi_{n m}\right\|_{m} \leq(2 e)^{n+m} C n ! R^{n+m} /(n+m) ! \\
& \leq C(2 e R)^{n+m} / m !,
\end{aligned}
$$

and hence $\Phi \in \mathcal{S}$.

Conversely, assume $\left(\varphi_{n}\right) \in \mathcal{S}$ and put $T_{n} \equiv H_{n} \varphi_{n}(D)$. We claim that $T_{n}$ satisfies the growth condition in Lemma 1 and hence that $\Phi(D)$ is a well defined continuous operator. This is easily checked in view of $T_{n} f(x)=$ $\left\langle f, \varphi_{n}\langle x,\rangle^{n} / n !\right\rangle$ and (2). It remains to prove that $T=\Phi(D)$ is PDE-preserving for the set of homogeneous polynomials. Thus assume $P \in \mathscr{P}\left({ }^{m} F\right)$ for some $m$. Now, $P(D) H_{n}=H_{n-m} P(D)$ if $n \geq m$, and $P(D) H_{n}=0$ otherwise. Further, $P(D)$ commutes with $\varphi_{n}(D)$ for all $n$. From this we deduce that $P(D) T=T^{(m)} P(D)$ where $T^{(m)} \equiv \Phi^{(m)}(D)$ and $\Phi^{(m)} \equiv$ $\left(\varphi_{m}, \varphi_{m+1}, \ldots\right) \in \mathcal{S}$. Hence $T \in \mathscr{O}(\mathbb{H})$.

We denote by $\mathscr{S}=\mathscr{S}(E, F)$ the set of functions $P \in \mathscr{H}_{\mathrm{G}}(E \times F)$ with the following property: $P(\cdot, y) \in \mathscr{H}_{\mathrm{Nb}}(E)$ for every fixed $y \in F$, and for every given $r>0$, there are constants $M=M_{r}, R=R_{r}>0$ such that $\|P(\cdot, y)\|_{\mathrm{N}: r} \leq M e^{R\|y\|}$. Thus $P(x, \cdot) \in \operatorname{Exp}(F)$ for every fixed $x \in E$.

Proposition 2. Every $P \in \mathscr{S}$ defines a continuous operator $P(\cdot, D)$ : $\mathscr{H}_{\mathrm{Nb}}(E) \rightarrow \mathscr{H}_{\mathrm{Nb}}(E)$ by $P(\cdot, D) f(x) \equiv\left\langle f, P(x, \cdot) e_{x}\right\rangle$. Conversely, for every continuous operator $T \in \mathscr{L}$ on $\mathscr{H}_{\mathrm{Nb}}(E)$ there is a unique symbol $P \in \mathscr{S}$ such that $T=P(\cdot, D)$, in fact, $P(x, y)=e^{-\langle x, y\rangle} T e_{y}(x)$. (Thus, $P(\cdot, D) Q(\cdot, D)=R(\cdot, D)$ where $\left.R(x, y)=e^{-\langle x, y\rangle}\left\langle Q(\cdot, y) e_{y}, P(x, \cdot) e_{x}\right\rangle \cdot\right)$

Proof. We prove that $P(\cdot, D), P \in \mathscr{S}$, defines a continuous operator on $\mathscr{H}_{\mathrm{Nb}}$. Let $f=\sum f_{n} \in \mathscr{H}_{\mathrm{Nb}}$, where $f_{n}=\sum_{k}\left\langle\cdot, y_{n k}\right\rangle^{n} \in \mathscr{P}_{\mathrm{N}}\left({ }^{n} E\right)$, be arbitrary. We note that $\left\langle f_{n}, P(x, \cdot) e_{x}\right\rangle=n ! \sum_{k} H_{n}\left(P(x, \cdot) e_{x}\right)\left(y_{n k}\right)$, and thus, pointwise,

$$
P(\cdot, D) f=\sum_{n} \sum_{k} \sum_{i+j=n} n ! P_{j}\left(\cdot, y_{n k}\right)\left\langle\cdot, y_{n k}\right\rangle^{i} / i !
$$

where $P_{n}(x, y) \equiv D_{y}^{n} P(x, \cdot)(0) / n$ !. Now $P_{j}\left(\cdot, y_{n k}\right)\left\langle\cdot, y_{n k}\right\rangle^{i} \in \mathscr{H}_{\mathrm{Nb}}$, and thus, to prove that $x \mapsto\left\langle f, P(x, \cdot) e_{x}\right\rangle \in \mathscr{H}_{\mathrm{Nb}}$, we only have to prove that the series (8) converges absolutely in $\mathscr{H}_{\mathrm{Nb}}$. If $\|P(\cdot, y)\|_{\mathrm{N}: r} \leq M_{r} e^{R_{r}\|y\|}, r>0$, 
we deduce from Cauchy's formula that $\left\|P_{n}(\cdot, y)\right\|_{\mathrm{N}: r} \leq M_{r} R_{r}^{n} e^{n}\|y\|^{n} / n$ ! for all $n$. Thus, by the binomial formula,

$$
T_{n k} \equiv \sum_{i+j=n}\left\|n ! P_{j}\left(\cdot, y_{n k}\right)\left\langle\cdot, y_{n k}\right\rangle^{i} / i !\right\|_{\mathrm{N}: r} \leq\left\|y_{n k}\right\|^{n} C_{r} N_{r}^{n}
$$

for some constants $C_{r}, N_{r}>0$ (independent of $f$ ). Hence $\sum_{n} \sum_{k} T_{n k} \leq$ $C_{r}\|f\|_{\mathrm{N}: N_{r}}$, and $f \mapsto P(\cdot, D) f$ is continuous.

We note that $P(x, y)=e^{-\langle x, y\rangle} P(\cdot, D) e_{y}(x)$ if $P \in \mathscr{S}$ and prove that $Q(x, y) \equiv T e_{y}(x) \in \mathscr{S}$ for any continuous operator $T$. Clearly, $Q(\cdot, y) \in$ $\mathscr{H}_{\mathrm{Nb}}$, and since $Q(x, y)={ }^{t} T e_{x}(y)$, we have $Q(x, \cdot) \in$ Exp. Next, by the continuity of $T$ and the fact that $\left\|e_{y}\right\|_{\mathrm{N}: r} \leq e^{r\|y\|}$, our claim follows. Hence, $Q \in \mathscr{S}$ and consequently, $P(x, y) \equiv e^{-\langle x, y\rangle} Q(x, y) \in \mathscr{S}$ and $P(\cdot, D) e_{y}=T e_{y}$ for all $y \in F$. Thus, by totality, $T=P(\cdot, D)$.

REMARK 1. Assume that $E$ and $F$ are finite-dimensional, i.e. $E=F=$ $\mathbb{C}^{d}$ for some $d$, and let $P(x, y)=\sum_{\alpha, \beta \in \mathbb{N}^{d}} P_{\alpha \beta} x^{\alpha} y^{\beta} \in \mathscr{S}$. Then $P(\cdot, D)$ is the infinite type differential operator $f \mapsto \sum P_{\alpha \beta} x^{\alpha} D^{\beta} f(x)$. Thus, for arbitrary $E$ and $F$, we may consider any element $P(\cdot, D)$ as an infinite type differential operator with variable coefficients, and Proposition 2 shows that every element of $\mathscr{L}$ is of this type.

Thus $\mathscr{C}(E)$ is the subring of $\mathscr{L}$ formed by the operators with symbols that do not depend on the first variable, and in view of Remark 1, we may consider any element of $\mathscr{C}(E)$ as an infinite type differential operator with constant coefficients.

We note that $\Phi(D) e_{y}(x)=\sum\langle x, y\rangle^{n} \varphi_{n}(y) / n$ ! for any $\Phi=\left(\varphi_{n}\right) \in \mathcal{S}$, hence the symbol for $\Phi(D)$ is given by $e^{-\langle x, y\rangle} \sum\langle x, y\rangle^{n} \varphi_{n}(y) / n$ !. Next we describe how to obtain these types of symbols, and thus $\mathscr{O}(\mathbb{H})$, in another way. For any $n \geq 0$ we let \langle\rangle$^{n} \in \mathscr{S}$ where \langle\rangle$^{n}(x, y) \equiv\langle x, y\rangle^{n}$.

TheOREM B. The ring $\mathscr{O}(\mathbb{H})$ is the subring of $\mathscr{L}$ formed by the operators $\Phi(\cdot, D)=\sum\left(\langle\rangle^{n}(\cdot, D) / n !\right) \phi_{n}(D)$, i.e. $\Phi(x, y) \equiv \sum \phi_{n}(y)\langle x, y\rangle^{n} / n ! \in \mathscr{S}$, where $\Phi=\left(\phi_{n}\right) \in \mathcal{S}$ and is unique. Further, $\Phi(D)=\sum\left(\langle\rangle^{n}(\cdot, D) / n !\right) \phi_{n}(D)$, $\Phi=\left(\varphi_{n}\right) \in \mathcal{S}$, where

$$
\varphi_{n}=\sum_{k=0}^{n}\left(\begin{array}{l}
n \\
k
\end{array}\right) \phi_{k}, \quad \phi_{n}=\sum_{k=0}^{n}\left(\begin{array}{l}
n \\
k
\end{array}\right)(-1)^{n-k} \varphi_{k} .
$$

Proof. Assume $\left(\phi_{n}\right) \in \mathcal{S}$ and define $\varphi_{n}$ by (9). Then it is easily checked that $\Phi=\left(\varphi_{n}\right) \in \mathcal{S}$ and $\Phi(x, y) \equiv \sum \phi_{n}(y)\langle x, y\rangle^{n} / n ! \in \mathscr{S}$. We prove that $\Phi(D)=\Phi(\cdot, D)$. We note that $H_{m}\langle\rangle^{n}(\cdot, D) / n !=\left(\begin{array}{c}m \\ n\end{array}\right) H_{m}$ if $n \leq m$, and $H_{m}\langle\rangle^{n}(\cdot, D)=0$ otherwise. Hence

$$
H_{m} \Phi(\cdot, D)=\sum_{n=0}^{\infty} H_{m} \frac{\langle\rangle^{n}(\cdot, D)}{n !} \phi_{n}(D)=\sum_{n=0}^{m}\left(\begin{array}{c}
m \\
n
\end{array}\right) H_{m} \phi_{n}(D)
$$


for all $m \geq 0$. Thus, for any $m \geq 0$,

$$
H_{m} \Phi(D)=H_{m} \varphi_{m}(D)=H_{m} \sum_{n=0}^{m}\left(\begin{array}{c}
m \\
n
\end{array}\right) \phi_{n}(D)=H_{m} \Phi(\cdot, D),
$$

hence $\Phi(D)=\Phi(\cdot, D)$. Conversely, let $\Phi(D) \in \mathscr{O}(\mathbb{H}), \Phi=\left(\varphi_{n}\right) \in \mathcal{S}$, be arbitrary. If $\phi_{n}$ is defined by the right hand equality in $(9)$, then $\left(\phi_{n}\right) \in \mathcal{S}$. The inversion formula on the left hand side in (9) holds true. Indeed,

$$
\begin{aligned}
\sum_{k=0}^{n} \sum_{m=0}^{k}\left(\begin{array}{l}
n \\
k
\end{array}\right)\left(\begin{array}{l}
k \\
m
\end{array}\right)(-1)^{k-m} \varphi_{m} & =\left.\sum_{k=0}^{n} \sum_{m=0}^{\infty}\left(\begin{array}{l}
n \\
k
\end{array}\right) \frac{\varphi_{m}}{m !} \frac{d^{m}}{d z^{m}} z^{k}\right|_{z=-1} \\
& =\left.\sum_{m=0}^{\infty} \frac{\varphi_{m}}{m !} \frac{d^{m}}{d z^{m}}(z+1)^{n}\right|_{z=-1}=\varphi_{n} .
\end{aligned}
$$

Hence, by the first part of the proof, $\Phi(D)=\Phi(\cdot, D)$, where $\Phi(x, y) \equiv$ $\sum \phi_{n}(y)\langle x, y\rangle^{n} / n ! \in \mathscr{S}$. Finally, the uniqueness follows from the uniqueness in Theorem $\mathrm{A}$ and the arguments above.

From the results above it follows that $P \in \mathscr{S}$ is a symbol for an operator in $\mathscr{O}(\mathbb{H})$ if and only if $e^{\langle x, y\rangle} P$ (or $e^{-\langle x, y\rangle} P$ ) is such a symbol.

We shall see that the operators in $\mathscr{O}(\mathbb{H})$ can also be expanded in powers of the Euler operator $\langle\cdot, D\rangle \equiv\langle\rangle(\cdot, D)$. We set

$$
E_{n m} \equiv D^{n}\left(e^{z}-1\right)^{m}(0) / n !=\sum_{i=0}^{m}\left(\begin{array}{c}
m \\
i
\end{array}\right)(-1)^{m-i} i^{n} / n !, \quad E \equiv\left(E_{n m}\right)
$$

(note that $E_{n m}=0$ if $n<m$ ). Given $n \leq m, S_{n m}$ denotes the set of all increasing sequences $s=\left\{k_{0}=n, k_{1}, \ldots, k_{l}=m\right\}, k_{i}<k_{i+1}$ and $s(E) \equiv(-1)^{l} E_{k_{l} k_{l-1}} \cdots E_{k_{2} k_{1}} E_{k_{1} k_{0}}$. Let $\Sigma_{n m}(E) \equiv \sum_{s \in S_{n m}} s(E)$ and hence, in particular, $\Sigma_{n n}(E) \equiv 1$. If $n>m$ we put $\Sigma_{n m}(E) \stackrel{\equiv}{\equiv}$. The elements $\Sigma_{n m}, m \geq n$, are uniquely determined by $\sum_{n=0}^{r} E_{n m} \Sigma_{n r}=\delta_{r}^{m}$.

Theorem C. $\mathscr{O}(\mathbb{H})$ consists of the operators $\sum\left(\langle\cdot, D\rangle^{n} / n !\right) \phi_{n}(D)$ where $\left(\phi_{n}\right) \in \mathcal{S}$ and is unique. Further, $\Phi(D)=\sum\left(\langle\cdot, D\rangle^{n} / n !\right) \phi_{n}(D), \Phi=\left(\varphi_{n}\right)$ $\in \mathcal{S}$, where

$$
\varphi_{n}=\sum_{k=0}^{\infty} \frac{n^{k}}{k !} \phi_{k}, \quad \phi_{n}=\sum_{k=0}^{\infty} \sum_{m=k}^{\infty}\left(\begin{array}{c}
m \\
k
\end{array}\right)(-1)^{m-k} \Sigma_{n m}(E) \varphi_{k} .
$$

Proof. It is easily checked that $\langle\cdot, D\rangle^{n}=\Psi_{n}(D)$ where $\Psi_{n}=\left(\psi_{k} \equiv k^{n}\right)$ $\in \mathcal{S}$, and hence that $f \mapsto \sum\left(\langle\cdot, D\rangle^{n} / n !\right) \phi_{n}(D) f$ defines a continuous operator $T$ on $\mathscr{H}_{\mathrm{Nb}}$ for any $\left(\phi_{n}\right) \in \mathcal{S}$. Every term $\left(\langle\cdot, D\rangle^{n} / n !\right) \phi_{n}(D)$ is in $\mathscr{O}(\mathbb{H})$ and hence, since $\mathscr{O}(\mathbb{H})$ is closed in $\mathscr{L}$ for the topology of pointwise convergence, $T \in \mathscr{O}(\mathbb{H})$. By Theorem $\mathrm{A}, T=\Phi(D)$ for some $\Phi=\left(\varphi_{n}\right) \in \mathcal{S}$. Now, $n ! H_{n} \Phi(D) e_{y}=\langle\cdot, y\rangle^{n} \varphi_{n}(y)$ and from $\langle\cdot, D\rangle^{n}=\Psi_{n}(D)$ we deduce 
$n ! H_{n} T e_{y}=\langle\cdot, y\rangle^{n} \sum_{k=0}^{\infty} \phi_{k}(y) n^{k} / k !$. Thus the left hand formula of (10) holds.

Next, let $T=\sum H_{n} \varphi_{n}(D)=\sum\left(\langle\rangle^{n}(\cdot, D) / n !\right) \psi_{n}(D)$ be an arbitrary operator in $\mathscr{O}(\mathbb{H})$. We claim: For every $r>0$ there are constants $R, C>0$ such that $\left|\Sigma_{n m}\right| \leq C R^{n} r^{m}$. Indeed, by Cauchy's formula, we have $\left|E_{n m}\right| \leq$ $\left(e^{r}-1\right)^{m} / r^{n}$ for any $r>0$. Thus, for any $s \in S_{n m}$, where $n \leq m$, and $r>0$,

$$
|s(E)| \leq \frac{\left(e^{r_{1}}-1\right)^{n}}{r_{l}^{m}}\left(\frac{e^{r_{l}}-1}{r_{l-1}}\right)^{k_{l}-1} \cdots\left(\frac{e^{r_{2}}-1}{r_{1}}\right)^{k_{1}} \leq\left(e^{r_{1}}-1\right)^{n}\left(\frac{r}{2}\right)^{m}
$$

if we choose $r_{l} \geq 2 / r$ and then $r_{l-1} \geq e^{r_{l}}-1, r_{l-2} \geq e^{r_{l-1}}-1, \ldots$ Now, $S_{n m}$ consists of $2^{m-n-1}$ elements if $m>n$ and hence our claim.

Thus for $\phi_{n} \equiv \sum_{m} \Sigma_{n m} \psi_{m}$ we have $\left(\phi_{n}\right) \in \mathcal{S}$ and, by Theorem B, $\phi_{n}$ is given by the right hand formula in (10). By the first part of the proof, $\sum\left(\langle\cdot, D\rangle^{n} / n !\right) \phi_{n}(D)$ defines an operator $S$ in $\mathscr{O}(\mathbb{H})$. We now prove that $T=S$. It suffices to prove that their symbols in $\mathscr{S}$ coincide. The symbol for $T$ is given by $\sum \psi_{m}(y)\langle x, y\rangle^{m} / m$ ! (Theorem B) and we deduce that $\sum_{m}\left(\sum_{n} E_{n m} \phi_{n}(y)\right)\langle x, y\rangle^{m} / m$ ! is the symbol for $S$. Thus, we only have to prove that $\psi_{m}=\sum_{n} E_{n m} \phi_{n}$. By our estimates for $\left|E_{n m}\right|$ and $\left|\Sigma_{n m}\right|$,

$$
\sum_{n} E_{n m} \phi_{n}=\sum_{n} \sum_{r} E_{n m} \Sigma_{n r} \psi_{r}=\sum_{r} \sum_{n} E_{n m} \Sigma_{n r} \psi_{r}=\sum_{r} \delta_{r}^{m} \psi_{r}=\psi_{m} .
$$

The uniqueness is a consequence of Theorem A and the above arguments.

4. The ring $\mathscr{O}(\mathbb{H}(\mathbb{P}))$. We have seen that the elements in $\mathscr{O}(\mathbb{H})$ can be identified with sequences of exponential type functions. This motivates the study of conditions on the sequence $\Phi$, for a given operator $\Phi(D) \in \mathscr{O}(\mathbb{H})$, in order that $\Phi(D) \in \mathscr{O}(\mathbb{H}(\mathbb{P}))$ where $\mathbb{H}(\mathbb{P}) \equiv \mathbb{H} \cup \mathbb{P}$ and $\mathbb{P} \subseteq$ Exp. Since $\mathscr{O}(\mathbb{H}(\mathbb{P}))=\bigcap_{\varphi \in \mathbb{P}} \mathscr{O}(\mathbb{H}(\varphi))$, it suffices to study $\mathscr{O}(\mathbb{H}(\varphi))$.

We let $Z(\varphi) \equiv\{a \in F: \varphi(a)=0\}$ denote the zero-set for $\varphi \in \operatorname{Exp}(F)$. If $a \in Z(\varphi)$ and $0 \neq b \in F$, we say that $a$ is a zero of order $M_{a}(b)$ in direction $b$ if $M_{a}(b)$ is the smallest integer $m \geq 0$ for which $D_{b}^{m} \varphi(a) \neq 0$. That is, the one-variable function $\varphi_{a: b}(z) \equiv \varphi(a+z b)$ has a zero at $z=0$ of order $M_{a}(b)$. Note that $M_{a}(b)=\infty$ if and only if $\varphi$ vanishes identically on the complex line $\{a+z b: z \in \mathbb{C}\}$. If $M_{a}(b)$ is finite we put $m_{a}(b) \equiv M_{a}(b)-1$.

It is well known that, for any $\varphi \in \operatorname{Exp}(F)$, the exponential polynomials $Q e_{a}$, where $a \in Z(\varphi)$ and $Q \in \mathscr{P}_{\mathrm{N}}\left({ }^{m} E\right)$ for some $m \geq 0$, in $\operatorname{ker} \varphi(D)$ form a total set in $\operatorname{ker} \varphi(D)$ (see [5]). In particular, $\langle\cdot, b\rangle^{m} e_{a} \in \operatorname{ker} \varphi(D)$ if $a \in Z(\varphi)$, $b \in F$ and $m<M_{a}(b)$, and it follows that, to obtain totality, it suffices to choose such exponential polynomials.

Lemma 3. Let $\phi \in \operatorname{Exp}(F)$. Then the set of exponential polynomials of the form $\langle\cdot, b\rangle^{m} e_{a}$, where $a \in Z(\phi), 0 \neq b \in F, M_{a}(b)<\infty$ and $m \leq m_{a}(b)$, is total in $\operatorname{ker} \phi(D)$. 
Proof. Assume $\varphi \in$ Exp is orthogonal to every such exponential polynomial. Now $\left\langle\langle\cdot, b\rangle^{m} e_{a}, \varphi\right\rangle=D_{b}^{m} \varphi(a)$, hence, for given $a \in Z(\phi)$ and $b$ with $M_{a}(b)<\infty, \varphi$ has a zero at $a$ of order $\geq M_{a}(b)$ in direction $b$. From this, and the arguments in the proof of Proposition 14 in [5], we deduce that $\varphi=\psi \phi$ for some exponential type function $\psi$. Thus if $f \in \operatorname{ker} \phi(D)$, then $\langle f, \varphi\rangle=\langle\phi(D) f, \psi\rangle=0$. Hence the lemma follows by virtue of the Hahn-Banach theorem.

We recall, from the proof of Theorem A, that if $m \geq 0$ and $T=\Phi(D) \in$ $\mathscr{O}(\mathbb{H})$ with $\Phi=\left(\varphi_{n}\right) \in \mathcal{S}$, then $T^{(m)} \equiv \Phi^{(m)}(D) \in \mathscr{O}(\mathbb{H})$ where $\Phi^{(m)} \equiv$ $\left(\varphi_{m}, \varphi_{m+1}, \ldots\right) \in \mathcal{S}$. Further, for any $a \in F$ and $m$ we write $D_{a}^{m} \Phi \equiv$ $\left(D_{a}^{m} \varphi_{n}\right) \in \mathcal{S}$ and $\Phi(a)$ is the sequence $\left(\varphi_{n}(a)\right)$ in $\mathbb{C}$.

THEOREM 1. Let $\phi=\sum \phi_{n}, \phi_{n} \in \mathscr{P}\left({ }^{n} F\right)$, be an exponential type function. An operator $T=\Phi(D), \Phi=\left(\varphi_{n}\right) \in \mathcal{S}$, is PDE-preserving for the set $\mathbb{H}(\phi)$ if the following holds true: For all $0 \neq a \in Z(\phi), 0 \neq b \in F$ with $M_{a}(b)<\infty$ and $m \leq m_{a}(b)$,

$$
D_{b}^{m}\left(\Phi^{(i)}-\Phi^{(j)}\right)(a)=0
$$

for all $i, j$ such that $\phi_{i}, \phi_{j} \neq 0$, i.e., $\varphi_{n+i}-\varphi_{n+j}$ has a zero at a of order $\geq M_{a}(b)$ in direction $b$ for all $n$.

Proof. By Lemma 3 we only have to prove that $\phi(D) T f=0$ where $f \equiv\langle\cdot, b\rangle^{m} e_{a} \in \operatorname{ker} \phi(D)$. Here $a \in Z(\phi), 0 \neq b \in F$ and $m \leq m_{a}(b)$. For any $\varphi \in \operatorname{Exp}$, we have $\varphi(D) f=e_{a} \sum_{i=0}^{m}\left(\begin{array}{c}m \\ i\end{array}\right)\langle\cdot, b\rangle^{i} D_{b}^{m-i} \varphi(a)$. Hence, if $a=0$, then $T f$ is spanned by $\langle\cdot, b\rangle^{j}, j \leq m$, and thus $T f \in \operatorname{ker} \phi(D)$. On the other hand, if $a \neq 0$, then by assumption, $\varphi_{n+i}(D) f=\varphi_{n+j}(D) f$ for all $n$ and $i, j$ such that $\phi_{i}, \phi_{j} \neq 0$. Thus, for any such $i$ and $j$, using $P(D) H_{n}=H_{n-m} P(D)$ for $n \geq m$ and $P \in \mathscr{P}\left({ }^{m} F\right)$, we obtain

$$
\begin{aligned}
\phi_{i}(D) T f & =\sum_{n \geq i} \phi_{i}(D) H_{n} \varphi_{n}(D) f=\sum_{n \geq i} H_{n-i} \phi_{i}(D) \varphi_{n}(D) f \\
& =\sum_{n \geq 0} H_{n} \phi_{i}(D) \varphi_{n+i}(D) f=\sum_{n \geq 0} H_{n} \phi_{i}(D) \varphi_{n+j}(D) f \\
& =\sum_{n \geq 0} H_{n} \varphi_{n+j}(D) \phi_{i}(D) f=T^{(j)} \phi_{i}(D) f .
\end{aligned}
$$

Hence, if $\phi_{j} \neq 0$, then $\phi(D) T f=T^{(j)} \phi(D) f=0$.

Example 1. Consider a continuous polynomial $P=1+P_{2}, P_{2} \in \mathscr{P}\left({ }^{2} F\right)$. Assume $T=\Phi(D)$ satisfies the condition in Theorem 1 and let $0 \neq a \in$ $Z(P)$. (Note that $-a \in Z(P)$, hence $\cosh \langle\cdot, a\rangle, \sinh \langle\cdot, a\rangle \in \operatorname{ker} P(D)$.) Then $\Phi(a)=(A, B, A, B, \ldots)$ and $T e_{a}=A \cosh \langle\cdot, a\rangle+B \sinh \langle\cdot, a\rangle$. If $2 \leq M_{a}(b)$ $<\infty$, then $D_{b} \Phi(a)=\left(D_{b} \varphi_{n}(a)\right)=(\alpha, \beta, \alpha, \beta, \ldots)$. We deduce that $T\langle\cdot, b\rangle e_{a}$ $=(A\langle\cdot, b\rangle+\alpha) \cosh \langle\cdot, a\rangle+(B\langle\cdot, b\rangle+\beta) \sinh \langle\cdot, a\rangle$. 
5. Ideal-preserving operators. We note that if $T \in \mathscr{O}(\mathbb{P})$, then ${ }^{t} T$ is ideal-preserving for the set $\mathbb{P}$ in the following sense:

Definition 2. A continuous linear operator $S: \operatorname{Exp} \rightarrow \operatorname{Exp}$ is idealpreserving for a set $\mathbb{P} \subseteq \operatorname{Exp}$ if $S \operatorname{Im} \varphi \subseteq \operatorname{Im} \varphi$ for every ideal $\operatorname{Im} \varphi=\operatorname{Exp} \cdot \varphi$, $\varphi \in \mathbb{P}$.

(Note that the set of ideal-preserving operators for a given set forms a ring.) Now, every ideal $\operatorname{Im} \varphi$ is closed $[3,5,6]$ and hence

(i) $\operatorname{Im} \varphi=\operatorname{ker} \varphi(D)^{\perp}$,

(ii) $[T \operatorname{ker} \varphi(D)]^{\perp}={ }^{t} T^{-1} \operatorname{Im} \varphi$,

and thus $T \in \mathscr{O}(\mathbb{P})$ implies ${ }^{t} T$ is ideal-preserving for $\mathbb{P}$. Conversely, if $S$ is ideal-preserving for $\mathbb{P}$ and if we assume that $S$ is the transpose of a continuous operator $T$ on $\mathscr{H}_{\mathrm{Nb}}$, i.e. $S$ is weakly continuous for the duality (1), then by (i) and (ii), $T \in \mathscr{O}(\mathbb{P})$. Thus if $E=F=\mathbb{C}^{d}$ for some $d$, then $\mathscr{H}_{\mathrm{Nb}}$ is reflexive and consequently every continuous operator on Exp is the transpose of a continuous operator on $\mathscr{H}_{\mathrm{Nb}}$; hence an operator is ideal-preserving for $\mathbb{P}$ if and only if it is the transpose of some $T \in \mathscr{O}(\mathbb{P})$. With somewhat different arguments we extend this result to arbitrary $E$ and $F$ in the case when $\mathbb{P} \supseteq \mathbb{H}$. (See also the proofs of Lemma 2 and Theorem A.)

TheOREM 2. An operator $S: \operatorname{Exp} \rightarrow \operatorname{Exp}$ is ideal-preserving for a set $\mathbb{P} \supseteq \mathbb{H}$ if and only if it is the transpose of some PDE-preserving operator $T \in \mathscr{O}(\mathbb{P})$.

Proof. In view of the discussion above, we only have to prove that if $S$ is ideal-preserving, then $S={ }^{t} T$ for some $T \in \mathscr{L}$. By assumption, for every $x \neq 0$ and $n \geq 0$ there is a unique $\varphi_{n: x} \in \operatorname{Exp}$ such that $\left[S\langle x, \cdot\rangle^{n} / n !\right](y)=$ $\varphi_{n: x}(y)\langle x, y\rangle^{n} / n !, y \in F$. Now, the left hand side is an $n$-homogeneous polynomial in $x$, and by homogeneity, we deduce that $\varphi_{n: x}$ does not depend on $x \neq 0$. Hence, for any $x \in E, S e_{x}=\sum S\langle x, \cdot\rangle^{n} / n !=\sum \varphi_{n}\langle x, \cdot\rangle^{n} / n$ ! for some sequence $\Phi \equiv\left(\varphi_{n}\right)$ in Exp. As in the proof of Theorem A, we deduce that $\Phi \equiv\left(\varphi_{n}\right) \in \mathcal{S}$ and note that $\left[S e_{x}\right](y)=\left[\Phi(D) e_{y}\right](x)=\left[{ }^{t} \Phi(D) e_{x}\right](y)$, i.e. by totality, $S={ }^{t} T, T=\Phi(D) \in \mathscr{L}$.

Thus, by Theorem 1 we obtain a sufficient condition for an operator to be ideal-preserving and are motivated to find the transposes of the operators in $\mathscr{O}(\mathbb{H})$. First, note that the transpose of any $\Phi(D) \in \mathscr{O}(\mathbb{H})$ is the operator $\sum \varphi_{n} H_{n}$, i.e., $\varphi \mapsto \sum \varphi_{n} H_{n}(\varphi)$ (see also $[8,10]$ ). Thus, in particular, an operator on Exp is ideal-preserving for $\mathbb{H}$ iff it has this form. Next, we show how to obtain the transpose when $T \in \mathscr{O}(\mathbb{H})$ is in the form of Theorem B. Note that the transpose of any $P(\cdot, D) \in \mathscr{L}, P \in \mathscr{S}$, is the operator $P(D, \cdot)$ defined by $\varphi \mapsto\left\langle P(\cdot, y) e_{y}, \varphi\right\rangle$. Hence, the transpose of any 
$\Phi(\cdot, D)=\sum\left(\langle\rangle^{n}(\cdot, D) / n !\right) \varphi_{n}(D) \in \mathscr{O}(\mathbb{H})$ is the operator $\sum \varphi_{n}\langle\rangle^{n}(D, \cdot) / n$ ! and, in particular, we obtain all ideal-preserving operators for $\mathbb{H}$ in this way.

In the proof of Theorem A we noted that if $P \in \mathscr{P}\left({ }^{m} F\right)$, then $P(D) T=$ $T^{(m)} P(D)$ for all $T \in \mathscr{O}(\mathbb{H})$. Thus, if $S$ is ideal-preserving for $\mathbb{H}$ and $P \in$ $\mathscr{P}\left({ }^{m} F\right)$, then $S P=P S^{[m]}$ where $S^{[m]} \equiv{ }^{t} T^{(m)}$ and $S={ }^{t} T$.

Since $\mathscr{H}_{\mathrm{Nb}}$ is Fréchet, an operator on $\mathscr{H}_{\mathrm{Nb}}$ is surjective iff its transpose is one-to-one and has a closed range for the duality (1). Further, every non-zero convolution operator on $\mathscr{H}_{\mathrm{Nb}}$ is surjective (Malgrange theorem) $[3,5,6]$. The analogue of the following result, when $E$ is finite-dimensional, is obtained in [10], and for a proof we refer to the arguments there. The statement gives examples of surjective operators in $\mathscr{O}(\mathbb{H}) \backslash \mathscr{C}$, and thus (Theorem 2) of injective closed-range ideal-preserving operators for $\mathbb{H}$.

Proposition 3. Let $\Phi=\left(\varphi_{n}\right)$ be a sequence of homogeneous polynomials $\varphi_{n} \in \mathscr{P}\left({ }^{n} F\right)$. Then $\Phi \in \mathcal{S}$ and $\Phi(D) \mathscr{H}_{\mathrm{Nb}}=\mathscr{H}_{\mathrm{Nb}}$ iff there are constants $M, N, R, r>0$ such that

$$
N r^{n} / n ! \leq\left\|\varphi_{n}\right\|_{n} \leq M R^{n} / n ! .
$$

(Thus if $\varphi \in \operatorname{Exp}, \varphi_{n} \equiv H_{n}(\varphi) \neq 0$ for all $n$ and $\liminf \left(\left\|\varphi_{n}\right\|_{n} n !\right)^{1 / n} \neq 0$, then $\Phi(D) \mathscr{H}=\mathscr{H}$.)

\section{References}

[1] J.-E. Björk, Analytic D-Modules and Applications, Kluwer, 1993.

[2] S. Dineen, Complex Analysis on Infinite Dimensional Spaces, Springer, 1999.

[3] T. A. W. Dwyer, Convolution equations for vector-valued entire functions of nuclear bounded type, Trans. Amer. Math. Soc. 217 (1976), 105-119.

[4] L. Filipsson, On polynomial interpolation and complex convexity, thesis, KTH, 1999.

[5] C. Gupta, Malgrange theorem for nuclearly entire functions of bounded type on a Banach space, Notas Mat. 37, Inst. Mat. Pura Apl., Rio de Janeiro, 1968.

[6] - , Convolution operators and holomorphic mappings on a Banach space, Sem. Anal. Mod. 2., Univ. Sherbrooke, Québec, 1969.

[7] H. Petersson, Interpolation spaces for PDE-preserving projectors on Hilbert-Schmidt entire functions, Rocky Mountain J. Math. 33 (2003).

[8] - The PDE-preserving operators on nuclearly entire functions of bounded type, Acta Math. Hungar. 100 (2003), 69-81.

[9] -, Kergin interpolation in Banach spaces, Studia Math. 153 (2002), 101-114.

[10] - Rings of PDE-preserving operators, to appear.

School of Mathematical Sciences

Chalmers/Göteborg University

Eklandagatan, SE-412 96 Göteborg, Sweden

E-mail: henripet@math.chalmers.se

Received January 27, 2003

Revised version December 10, 2003 